\title{
EL DEDUCIBLE EN EL SEGURO DE RESPONSABILIDAD CIVIL EN COLOMBIA*
}

DEDUCTIBLE IN LIABILITY INSURANCE IN COLOMBIA

\author{
MARÍA CRISTINA ISAZA POSSE** \\ Fecha de recepción: 19 de mayo de 2021 \\ Fecha de aceptación: 30 de mayo de 2021 \\ Disponible en línea: 30 de junio de 2021
}

Para citar este artículo/To cite this article

Isaza Posse, María Cristina. El deducible en el seguro de responsabilidad civil en Colombia,

54 Rev.Ibero-Latinoam.Seguros, 147-162 (2021).

https://doi.org/10.11144/Javeriana.ris54.dsrc

doi:10.11144/Javeriana.ris54.dsrc

\footnotetext{
* Artículo de reflexión

** Abogada Universidad Javeriana. Especialista y Magistra en Derecho de Seguros, Universidad Javeriana. Asesora y Consultora. Profesora Universitaria. Contacto: mcisaza@isazaposse.com
} 


\section{RESUMEN}

Se realiza una revisión del concepto de deducible en el contrato de seguro desde el punto de vista general, para luego precisar su aplicación, tratándose del seguro de responsabilidad civil en particular.

Palabras Clave: Deducible, franquicia, seguro de responsabilidad civil

\section{ABSTRACT}

A revision of the deductible concept is done on the insurance contract, from a general point of view. Then the application of this concept is narrowed down to the topic of Liability insurance.

Keywords: Deductible, franchise, liability insurance

\section{SUMARIO}

Introducción. Noción. La finalidad del deducible o la franquicia. El deducible en los seguros de daños reales. El deducible en los seguros de daños patrimoniales. La Jurisprudencia. Bibliografía. 


\section{INTRODUCCIÓN}

Para estudiar el tema del deducible en el Seguro de Responsabilidad Civil, procederemos a revisar primero el tema desde el punto de vista general, para luego abordar lo tocante a su aplicación cuando del seguro de responsabilidad civil se trata.

\section{NOCIÓN}

En el volumen V del Tratado de Derecho Comercial. Ernesto Eduardo Martorell (Dir), 2010, encontramos la noción del deducible, como sigue ${ }^{1}$ :

"Hemos visto precedentemente que el asegurador debe indemnizar a su asegurado por el daño que éste ha sufrido en su interés asegurado a consecuencia de un siniestro cubierto por la póliza, dentro de los alcances, límites y condiciones establecidas en la misma.

Y uno de esos límites a la obligación del asegurador de indemnizar el daño sufrido por su asegurado es el que resulta de la incorporación dentro de la respectiva póliza de seguro de una franquicia (28), es decir, de un importe o una parte del daño que, en el evento de ocurrir un siniestro amparado por la misma, deberá ser tomado en cuenta para determinar si el asegurado tendrá derecho a ser indemnizado -franquicias condicionales o simples- o para fijar el monto de la indemnización en caso de que lo tenga-franquicias incondicionales, absolutas o deducibles- (29).

Las razones por las cuales los aseguradores suelen incorporar en sus pólizas las llamadas franquicias, básicamente, son dos. Una es la eliminación de la cobertura que otorga el asegurador en sus pólizas de todos los siniestros de poca monta los que, a través de su frecuencia siniestral, lo pueden hacer incurrir en gastos e administración de importancia, que en algunos casos, podrían superar los daños sufridos por los intereses asegurados; la otra razón es inducir a los asegurados a que actúen con la mayor diligencia y prudencia posible en evitar la ocurrencia de siniestros, ya que imponer una parte de los daños a su cargo lo obligan a extremar las precauciones para evitar que ellos ocurran y también para limitar sus consecuencias (30).

Sin embargo, son los mismos asegurados, en muchos casos grandes asegurados que cuentan con una importante capacidad económica y financiera y también de prevención de siniestros, quienes a través de franquicias en sus contratos de seguros buscan una apreciable reducción en las primas que deben abonar a sus aseguradores. En estos casos ya no es el asegurador quien busca protegerse de una frecuencia de siniestros de poca monta o de estimular la conducta antisiniestral de su asegurado, sino es este último quien, por meras razones de economía, acuerda con su asegurador determinadas coberturas con franquicias que, en ciertos casos, pueden tener alguna importancia, reduciendo de esa forma el costo de la prima que debe pagarle.

1 Tratado de Derecho Comercial. Ernesto Eduardo Martorell (Dir), 2010. Vol. V. Buenos Aires. Argentina: Editorial La Ley, pp. 363 a 365. 


\section{FRANQUICIAS, CLASES DE FRANQUICIAS Y EL LLAMADO DESCUBIERTO OBLIGATORIO}

Como ya lo adelantáramos, tanto en los mercados de seguros como en los de reaseguro se utilizan básicamente dos clases o tipos de franquicias: las condicionales o simples y las incondicionales, absolutas o deducibles.

En las primeras, es decir las condicionales o simples, el asegurador indemnizará el daño sufrido por el interés asegurado cubierto por la póliza que haya emitido cuando el mismo se encuentre amparado por dicha póliza y además su monto exceda del importe o porcentaje establecido en la misma como franquicia, en cuyo caso paga el total del daño. (31)

En las segundas, esto es en las incondicionales o absolutas, también conocidas como franquicias deducibles, el asegurador indemnizará los daños verificados en el interés asegurado a consecuencia de un siniestro cubierto por la póliza cuando dichos daños superen la franquicia establecida en la misma, pero de la indemnización a pagar se deducirá el importe de dicha franquicia. (32)

Algunos autores, además de estos dos tipos de franquicias que hemos visto precedentemente, han conceptualizado lo que ellos llaman descubierto obligatorio, que sería cuando el asegurado conserva a su cargo una cierta parte de daño, con independencia de su intensidad y extensión. (33)

En nuestra opinión la franquicia deducible y el descubierto obligatorio, si bien se presentan como dos institutos distintos, al final del día sus efectos y consecuencias se presentan de alguna manera como similares desde el momento en que en ambos casos se ponen a cargo del asegurado una parte del daño sufrido por el interés asegurado a consecuencia de un siniestro cubierto por la póliza que ha contratado con su asegurador. (34)

En efecto, el descubierto obligatorio está vinculado con el infraseguro, es decir, el caso en que la suma asegurada acordada en la póliza como límite de responsabilidad del asegurador -según lo establece el citado artículo 61 de la Ley de Seguros- es inferior al valor total del interés asegurable, situación que da lugar a la aplicación de la llamada Regla Proporcional, con lo cual una parte del eventual daño sufrido por dicho interés terminará siendo del asegurado (35); en cambio en la franquicia deducible puede ocurrir que exista o no un infraseguro pero, de todas formas, el asegurado terminará soportando una parte del daño sufrido en su interés asegurado que se corresponda con el importe o el porcentaje de tal franquicia. (36)

En el Derecho Inglés existe lo que se denomina franchise, que podríamos equiparar a lo que se denomina franquicia simple y según la cual el asegurador solamente indemnizará el daño sufrido por el interés asegurable cuando el mismo exceda de la franchise establecido en la póliza; pero también existe el llamado excess o deductible, que sería el equivalente al nuestra franquicia deducible, según el cual el asegurado soporta la pérdida sufrida en el interés asegurable hasta el importe establecido en la póliza por tal concepto y a partir de allí, el asegurador indemniza exceso del mismo (37)". 
En Colombia dispone el artículo 1079 del Código de Comercio: "El asegurador no estará obligado a responder sino hasta concurrencia de la suma asegurada, sin perjuicio de lo dispuesto en el inciso segundo del artículo 1074”.

Conforme a lo dispuesto por el artículo 1103 del Código de Comercio "Las cláusulas según las cuales el asegurado debe soportar una cuota en el riesgo o en la pérdida, o afrontar la primera parte del daño, implican, salvo estipulación en contrario, la prohibición para el asegurado de protegerse respecto de tales cuotas, mediante la contratación de un seguro adicional. La infracción de esta norma producirá la terminación del contrato original”.

El profesor J. Efrén Ossa explica en su obra sobre la franquicia o deducible:

"Es la primera parte del daño que, fatal o eventualmente, ha de soportar el asegurado en virtud de la expresa estipulación del contrato.

Es deducible cuando sea cual fuere la extensión del daño (fatalmente) el asegurado debe cargar con la primera parte de él, y el asegurador con el excedente". ${ }^{2}$

De otro lado en el Diccionario de Seguros Mapfre (páginas 117,118, 169 y 170) se consigna:

"deducible. Cantidad o porcentaje establecido en una póliza cuyo importe ha de superarse para que se pague una reclamación. Es también sinónimo de franquicia.

"franquicia. Cantidad por la que el asegurado es propio asegurador de sus riesgos y en virtud de la cual, en caso de siniestro, soportará con su patrimonio la parte de los daños que le corresponda. ...

Si el importe del siniestro es inferior a la cantidad estipulada como franquicia, su coste correrá por completo a cargo del asegurado, si es superior, la aseguradora solo indemnizará por el exceso de aquella".

La jurisprudencia arbitral colombiana se ha referido al deducible de la siguiente manera: ${ }^{3}$

“3.5.3. El deducible en el contrato de seguro

El artículo 1079 del Código de Comercio establece que “el asegurador no estará obligado a responder sino hasta concurrencia de la suma asegurada, sin perjuicio de lo dispuesto en el inciso segundo del artículo 1074”. De allí se desprende que, en primer lugar, la responsabilidad del asegurador está limitada, cuantitativamente, al monto de la suma asegurada. Sin embargo, en ejercicio de la autonomía privada, también es posible pactar que el asegurado asuma un porcentaje o una cuota del riesgo que pretende trasladar a la aseguradora. Las convenciones en ese sentido se denominan

\footnotetext{
2 Ossa Gómez J. Efrén. Teoría General del Seguro. El Contrato. Bogotá Colombia: Editorial Temis, 1991. Segunda Edición actualizada, pp. 245 y 246.

3 Laudo Arbitral. Colpensiones contra AXA Colpatria Seguros S.A. Diciembre 22 de 2020. Árbitros: Arturo Solarte Rodríguez; Juan Carlos Esguerra Portocarrero; Alejandro Venegas Franco.
} 
"deducible" y se encuentran definidas en el artículo 1103 del Código de Comercio como las "cláusulas según las cuales el asegurado debe soportar una cuota en el riesgo o en la pérdida, o afrontar la primera parte del daño". Se trata, entonces, de la participación del asegurado en la asunción de los efectos patrimoniales del siniestro, que puede pactarse como una suma fija o como un porcentaje del valor asegurado. Como su nombre lo indica tal importe será "deducido" de la suma que la aseguradora debe reconocerle al asegurado, puesto que está a cargo de este último.

La doctrina ha definido el deducible como "la primera parte de la pérdida que el asegurado asume sobre el monto indemnizable de un siniestro. Puede consistir en una suma fija o en un porcentaje del quantum de la indemnización o en una combinación de ambos". ${ }^{4}$ En este sentido opera como un mecanismo para compartir los riesgos entre la aseguradora y el asegurado, quien deberá soportar una porción de la pérdida. En consecuencia, en cada caso habrá que analizar el pacto de las partes para determinar el valor efectivo que la aseguradora debe pagar, luego de aplicar el deducible correspondiente".

\section{LA FINALIDAD DEL DEDUCIBLE O LA FRANQUICIA}

Como se señaló el propósito buscado mediante la estipulación de un deducible en un contrato de seguro, en virtud del cual la primera parte del daño o la pérdida debe ser asumida por el asegurado, no es otra que la de mantener el interés y el esfuerzo del asegurado dirigido que la pérdida o el siniestro no ocurra.

La búsqueda de esta finalidad está implícita en texto del artículo 1103 del Código de Comercio cuando establece la prohibición de proteger esta parte del riesgo o de la pérdida mediante la contratación de un seguro adicional, salvo que medie estipulación en contrario.

El deducible implica que el asegurado soporta una parte o porcentaje estipulado de su propia pérdida, lo cual significa, que éste debe aplicarse o deducirse del valor de la pérdida del asegurado. Resultaría impensable que el deducible tenga como efecto que el asegurado asuma parte de la obligación a cargo de la aseguradora establecida de conformidad con lo pactado en el contrato de seguro.

"En la franquicia deducible el asegurador solo indemniza si el daño verificado excede la franquicia establecida en la póliza y cuando ello ocurre, es decir, si el daño supera tal franquicia, entonces el asegurador indemnizará el daño, pero deduciendo el monto de dicha franquicia. Conf. HalPerin -Morandi Op. cit. p. 558 y Barbato Y MeiliJ. Op. cit. pp. 261 y ss"5.

Para entender de manera más clara las dificultades que plantean con el cálculo del deducible en los contratos de seguro de responsabilidad civil, conviene hacer una

\footnotetext{
4 NARváez Bonnet, Jorge Eduardo. El contrato de seguro en el sector financiero. Tercera edición. Bogotá: Ed. Grupo Editorial Ibáñez, 2014, p.373

5 Tratado de Derecho Comercial. Ernesto Eduardo Martorell (Dir), 2010. Volumen V. Buenos Aires. Argentina: Editorial La Ley, p. 363.
} 
breve revisión de la clasificación de los contratos de seguro según la naturaleza del riesgo amparado.

Los seguros se clasifican atendiendo a la naturaleza del riesgo amparado en seguros de personas y seguros de daños.

Los primeros cubren al asegurado de aquellos riesgos que afectan la integridad corporal o física de la persona. "Garantizan el pago de un capital o una renta cuando se produce un hecho que afecta la existencia, salud o vigor del asegurado; generalmente terminan en un pago en dinero, pero las prestaciones están subordinadas a hechos atinentes directamente a la persona del asegurado"6.

Los seguros de daños, implican la protección contra un riesgo de carácter patrimonial, en sentido económico-jurídico. Se busca resarcir el daño patrimonial causado por la realización del riesgo. Los seguros de daños a su vez, se clasifican en dos: reales y patrimoniales.

1. Seguros Reales: "son aquellos que versan sobre cosas muebles o inmuebles, determinadas o determinables, amenazadas en su integridad física o en la integridad jurídica de los derechos radicados en ellas." Se conocen como seguros de "valor asegurado", en cuanto la determinación previa del valor del interés reviste especial importancia para delimitar la responsabilidad asumida por la aseguradora.

Como características propias de esta clase de seguros, anota el profesor Efrén Ossa, las siguientes:

a. El valor asegurado tiene un límite que le es propio, dado por el valor económico de la cosa amenazada o del interés jurídico a ella vinculado. El interés asegurable es siempre susceptible de ser estimado en dinero de manera previa al momento de celebrarse el contrato de seguro.

b. Como consecuencia de lo anterior, en esta clase de seguros son aplicables los principios del sobreseguro y del infraseguro, en los casos en que se presenten diferencias entre el valor declarado por el tomador y el valor real del interés.

c. Se admite la procedencia del valor presunto (terrestres) y del valor estimado (marítimo).

d. Dada su naturaleza, admiten también, la viabilidad del seguro de valor a nuevo, ya que con ello se busca reponer o reemplazar la cosa asegurada en caso de pérdida.

2. Seguros Patrimoniales: "Como su nombre lo indica, protegen la integridad del patrimonio económico contra el detrimento eventual que pueda afectarlo desfavorablemente y que tanto puede originarse en una disminución del activo como en un aumento del pasivo". Estos se conocen como "seguros de suma", en cuanto la responsabilidad de la aseguradora se circunscribe al monto de la suma establecida como límite para tal efecto.

6 HalPerin, Isaac. Seguros. Buenos Aires: Ediciones Depalma, 1970, p. 8.

7 Ossa Gómez, J. Efrén. Teoría General del Seguro. El Contrato. Bogotá. Colombia: Editorial Temis, 1991, p. 61.

8 Ossa Gómez, J. Efrén. ob. cit., p. 63. 
Anota el mismo autor, la dificultad que se encuentra para delimitar el ámbito de los seguros patrimoniales y para subrayar sus diferencias con los seguros reales, habida consideración de que todos los seguros de daños tienen, en últimas la misma función. Señala entonces, que el procedimiento a seguir será el de analizar cada póliza para desentrañar así, la ubicación que le conviene en cada caso.

Sin embargo, anota el profesor Ossa, como características propias del seguro de responsabilidad civil extracontractual, cuya naturaleza es claramente patrimonial, las siguientes:

a. El interés asegurable no soporta una estimación previa en dinero. Su cálculo es aproximado, su suficiencia o insuficiencia solo se determina una vez se conozca la magnitud real del daño causado por el siniestro.

b. La fijación o determinación de la suma asegurada se deja al arbitrio de las partes contratantes, es de carácter netamente convencional.

c. Los conceptos de sobreseguro e infraseguro no son compatibles con la naturaleza jurídica de esta clase de seguros.

d. La subrogación del asegurador solo tiene cabida por vía de excepción.

Es procedente resaltar que, en los seguros reales, el detrimento patrimonial se origina con ocasión del acaecimiento del siniestro o, lo que es lo mismo, con ocasión de la realización del riesgo previsto, por la pérdida relacionada con un bien mueble o inmueble. Por el contrario, en los seguros patrimoniales la realización del riesgo afecta el patrimonio del asegurado considerado como una universalidad, sin que la pérdida sufrida con ocasión del siniestro guarde una relación directa con un bien concreto, mueble o inmueble.

\section{EL DEDUCIBLE EN LOS SEGUROS DE DAÑOS REALES}

En los seguros de daños reales, el deducible se aplica sobre el valor de la pérdida sufrida en el bien asegurado, lo cual se refleja de la misma manera en el valor de la indemnización, que se calcula o establece sobre el valor asegurado.

Ejemplo 1.A:

Pérdida total:

Valor bien: $\$ 1.000$

Valor asegurado: $\$ 1.000$

Deducible: $10 \%$ sobre el valor de la pérdida

Siniestro:

Valor pérdida: $\$ 1.000$

Menos 10\% deducible: $\$ 100=\$ 900$

Valor indemnización: $\$ 900$

En este caso, como el valor de la pérdida coincide con el valor asegurado, al aplicar el porcentaje al valor de la pérdida arroja el mismo resultado que si aplicamos el porcentaje pactado como deducible al valor asegurado. 
Ejemplo 1.B:

Pérdida total:

Valor bien: $\$ 1.000$

Valor asegurado: $\$ 1.000$

Deducible: $\$ 100$

Siniestro:

Valor pérdida: $\$ 1.000$

Menos deducible: $\$ 100=\$ 900$

Valor indemnización: $\$ 900$

En este caso, como el valor de la pérdida coincide con el valor asegurado, al restar la suma pactada como deducible arroja el mismo resultado que si restamos la suma pactada como deducible al valor asegurado.

Ejemplo 2.A:

Pérdida parcial:

Valor bien: $\$ 1.000$

Valor asegurado: $\$ 1.000$

Deducible: $10 \%$ sobre el valor de la pérdida

Siniestro:

Valor pérdida: $\$ 500$

Menos 10\% deducible: $\$ 50=\$ 450$

Valor indemnización: $\$ 450$

En este supuesto el valor del deducible es inferior al 10\% del valor asegurado.

Ejemplo 2.B:

Pérdida parcial:

Valor bien: $\$ 1.000$

Valor asegurado: $\$ 1.000$

Deducible: 100

Siniestro:

Valor pérdida: $\$ 500$

Menos deducible: $\$ 100=\$ 400$

Valor indemnización: $\$ 400$

En este caso el valor del deducible no guarda una relación porcentual con la pérdida, es una cantidad previamente acordada.

En el evento de que se presente un infraseguro, se aplicará primero el infraseguro o regla proporcional para determinar el valor de la pérdida indemnizable y luego se resta el deducible para establecer el valor de la indemnización a cargo de la aseguradora. 
"Cuando hay un infraseguro hay un descubierto, es decir, que una parte del daño va a ser soportada exclusivamente por el asegurado, quien de esa forma lo termina compartiendo con su asegurador en proporción a sus respectivos intereses. En cambio, cuando hay una franquicia deducible, si bien también el asegurado va a terminar soportando una parte del daño sufrido por el interés asegurado, el origen de ello no va a ser consecuencia de un infraseguro que puede existir o no, sino de lo que las partes voluntariamente hayan acordado. Podría ocurrir que en un seguro haya un infraseguro y que además incluya una franquicia deducible y entonces en este caso, primero habrá que determinar el monto de la indemnización que estaría a cargo de la póliza teniendo en cuenta dicho infraseguro y una vez obtenido ese importe, habría que restarle el importe de la franquicia deducible para, de esa forma, establecer el valor de la indemnización del asegurador; como se comprenderá en este caso, el asegurado terminará soportará (sic) dos deducciones: una proveniente del infraseguro y otra de la franquicia deducible". ${ }^{9}$

Como puede apreciarse con claridad, el deducible pactado, bien sea que la pérdida sea total o parcial, se calcula aplicando el porcentaje al valor de la pérdida sufrida por el interés asegurado o deduciendo la suma acordada del valor de dicha la pérdida. Una vez restado el valor correspondiente al deducible, se determina el alcance de la indemnización a cargo de la aseguradora.

La obligación de la aseguradora de indemnizar no será en ningún caso, equivalente al valor asegurado total en los seguros de daños reales en los que se ha pactado un deducible. No ocurre lo mismo con los seguros de daños patrimoniales.

\section{EL DEDUCIBLE EN LOS SEGUROS DE DAÑOS PATRIMONIALES}

En los seguros de daños patrimoniales, la pérdida no se concreta sobre un bien en particular, sino sobre el patrimonio concebido como universalidad. Dentro de esta categoría se encuentra el seguro de responsabilidad civil.

"Tratándose de seguro de daños, en los términos del artículo 1083 del Código de Comercio, tiene interés asegurable toda persona cuyo patrimonio pueda resultar afectado, directa o indirectamente, por un riesgo. Como acertadamente lo ha señalado la doctrina el asegurado es el titular del interés asegurable y, por consiguiente, la persona protegida. ${ }^{10}$

Tratándose del seguro de responsabilidad civil:

"Dado que el seguro de responsabilidad tiene por objeto, además de proteger a la víctima, salvaguardar el patrimonio del potencial responsable (quien es el asegurado),

\footnotetext{
9 Tratado de Derecho Comercial. Ernesto Eduardo Martorell (Dir), 2010. Volumen V. Buenos Aires. Argentina: Editorial La Ley, p. 365.

10 Ossa Gómez, J. Efrén, Teoría general del seguro. El contrato. Editorial Temis, Bogotá, 1991, p. 9.
} 
el interés se circunscribe a la preservación de dicho patrimonio que es susceptible de erosionarse por deudas de responsabilidad"11.

"Es necesario establecer el valor real del interés asegurado, lo cual se traduce en la suma asegurada, que limita la responsabilidad máxima de la compañia de seguros (artículo 1079 del Código de Comercio)"'12.

La suma asegurada bajo esta clase de seguros establece el límite máximo por el cual responde la aseguradora. No cabe entonces, tomando en consideración la naturaleza del riesgo asegurable, establecer la suma asegurada con referencia a un valor asegurable. En consecuencia, como lo afirma el profesor Ossa, las figuras del infraseguro y de sobreseguro resultan incompatibles con la naturaleza de los seguros de daños patrimoniales como son los seguros de responsabilidad civil. Podemos hablar de suficiencia e insuficiencia de la suma asegurada pero no de sobreseguro e infraseguro.

El deducible en esta clase de seguros, al igual que en toda clase de contrato de seguro se establece aplicando el porcentaje pactado o restando el valor pactado como franquicia del valor de la pérdida sufrida en el patrimonio del asegurado.

Ejemplos:

- 1.A. Pérdida por valor equivalente a la suma asegurada:

Valor o suma asegurada: $\$ 1.000$

Deducible: $10 \%$ sobre el valor de la pérdida

Siniestro:

Valor de la pérdida: $\$ 1.000$

Menos 10\% deducible: $\$ 100=\$ 900$

Valor indemnización a cargo de la aseguradora: $\$ 900$

En este caso aplicando el porcentaje del deducible pactado al valor de la pérdida arroja el mismo resultado que si lo aplicásemos a la suma asegurada.

- 1.B. Pérdida por valor equivalente a la suma asegurada:

Valor o suma asegurada: $\$ 1.000$

Deducible: $\$ 100$

Siniestro:

Valor de la pérdida: $\$ 1.000$

Menos deducible: $\$ 100=\$ 900$

Valor indemnización a cargo de la aseguradora: $\$ 900$

\footnotetext{
11 Diaz-Granados Ortiz, Juan Manuel. El seguro de responsabilidad. Bogotá D.C.: Editorial Universidad del Rosario y Pontificia Universidad Javeriana, Facultad de Ciencias Jurídicas. $2^{\circ}$ Edición, 2012.

12 Diaz-Granados Ortiz, Juan Manuel. El seguro de responsabilidad. Bogotá D.C.: Editorial Universidad del Rosario y Pontificia Universidad Javeriana, Facultad de Ciencias Jurídicas. $2^{\circ}$ Edición. 2012.
} 
En este caso descontando deducible pactado al valor de la pérdida arroja el mismo resultado que si lo aplicásemos a la suma asegurada.

- 2.A. Pérdida por valor inferior a la suma asegurada:

Valor o suma asegurada: $\$ 1.000$

Deducible: $10 \%$ sobre el valor de la pérdida

Siniestro:

Valor pérdida: $\$ 500$

Menos 10\% deducible: $\$ 50=\$ 450$

Valor indemnización a cargo de la aseguradora: $\$ 450$

En este supuesto el valor del deducible aplicado sobre el valor de la pérdida es inferior al $10 \%$ de la suma asegurada.

- 2.B. Pérdida por valor inferior a la suma asegurada:

Valor o suma asegurada: $\$ 1.000$

Deducible: $\$ 100$

Siniestro:

Valor pérdida: $\$ 500$

Menos $\$ 100$ deducible: $\$ 100=\$ 400$

Valor indemnización a cargo de la aseguradora: $\$ 400$

En este supuesto el valor del deducible será restado del valor de la pérdida.

- 3.A. Pérdida por valor superior a la suma asegurada:

Valor o suma asegurada: $\$ 1.000$

Deducible: $10 \%$ sobre el valor de la pérdida

Siniestro:

Valor pérdida: $\$ 2.000$

Menos 10\% deducible: $\$ 200=\$ 1.800$

Valor indemnización a cargo de la aseguradora: $\$ 1.000$

Correrán por cuenta del asegurado los primeros $\$ 200$ de la pérdida correspondientes al deducible y los $\$ 800$ que van en exceso de la cobertura estipulada.

- 3.B. Pérdida por valor superior a la suma asegurada:

Valor o suma asegurada: $\$ 1.000$

Deducible: $\$ 100$

Siniestro:

Valor pérdida: $\$ 2.000$

Menos 10\% deducible: $\$ 100=\$ 1.900$

Valor indemnización a cargo de la aseguradora: $\$ 1.000$ 
La aseguradora responderá hasta el monto de la suma asegurada estipulada. Correrán por cuenta del asegurado los primeros $\$ 100$ de la pérdida correspondientes al deducible y los $\$ 900$ que van en exceso de la cobertura estipulada.

En el supuesto de que la pérdida sufrida por el asegurado supere la suma asegurada, la aseguradora responderá por toda la suma asegurada estipulada en el contrato de seguro de responsabilidad civil, la cual constituye el límite máximo de su responsabilidad. El deducible se aplicará descontándolo del valor de la pérdida, no de la suma asegurada pactada.

Resulta relevante respecto a este punto la prohibición prevista en el artículo 1103 del Código de Comercio, donde establece “...el asegurado debe soportar una cuota en el riesgo o en la pérdida, o afrontar la primera parte del daño, implican, salvo estipulación en contrario, la prohibición para el asegurado de protegerse respecto de tales cuotas, mediante la contratación de un seguro adicional". Si se aceptarse la tesis de que el deducible se aplica sobre la suma asegurada o sobre el valor de la indemnización a cargo de la aseguradora, y no sobre la pérdida, en el caso de contratarse un seguro adicional, estaríamos, en caso de subsistir los dos contratos frente a una concurrencia o coexistencia de seguros, no de un deducible o asunción de una cuota del riesgo o de la pérdida por parte del asegurado. Se trata de una parte de la pérdida no cubierta, razón por la cual la sanción prevista para su inobservancia es la terminación del contrato de seguro original y en caso de contarse con la autorización de la aseguradora existen dos contratos de seguro independientes que amparan partes del riesgo diferentes.

La prohibición se refiere a la cobertura del deducible, de la parte del riesgo o parte de la pérdida que debe soportar el asegurado. En consecuencia, el concepto del deducible es la parte del riesgo que soporta el asegurado, que no es materia de cobertura mediante el contrato de seguro y no hace parte de la obligación condicional de la aseguradora exigible con la ocurrencia del siniestro. Lo anterior lleva a concluir que el deducible no puede aplicarse a la suma asegurada estipulada, sino al valor de la pérdida sufrida por el asegurado.

Aceptar la postura adoptada por algunos cuando el valor de la pérdida supera la suma asegurada, descontando el deducible del valor final de la indemnización a su cargo, que es la suma asegurada, traería como consecuencia, que entre mayor sea el deducible o la franquicia estipulada, menor será la cobertura real ofrecida por la aseguradora.

El tomador asegurado suscribe el contrato de seguro de responsabilidad civil bajo el entendido de que la aseguradora responderá hasta por la suma asegurada pactada, suma sobre la cual se determina el valor de la prima. La metodología usada en la que se aplica el deducible sobre la suma asegurada en los casos en que el valor de la pérdida supera dicha suma asegurada constituye una abierta violación de los términos y condiciones estipulados en el contrato de seguro de responsabilidad.

El deducible implica que el asegurado soporta una parte o porcentaje estipulado de su propia pérdida, lo cual significa, que SIEMPRE este debe aplicarse o deducirse, del valor de la pérdida del asegurado. Resulta impensable que el deducible tenga 
como efecto que el asegurado asuma parte de la obligación a cargo de la aseguradora establecida de conformidad con lo pactado en el contrato de seguro.

Cuando la suma asegurada pactada es insuficiente de cara a la pérdida sufrida, el asegurado deberá soportar además del deducible estipulado calculado sobre el valor de la pérdida, el valor que vaya en exceso de la suma asegurada o cobertura pactada.

\section{LA JURISPRUDENCIA}

A manera de ejemplo de la forma como usualmente la jurisprudencia y las aseguradoras aplican el deducible tratándose del seguro de responsabilidad civil, me permito citar la parte correspondiente de la siguiente sentencia.

La Corte Suprema de Justicia en sentencia sustitutiva de diciembre de 2019 adoptó una decisión condenatoria a la aseguradora, en el sentido que se pasa a señalar:

"Dentro de los términos y condiciones del contrato de seguro, cabe destacar los siguientes: (i) amparo del riesgo del deterioro patrimonial, como consecuencia de tos errores y omisiones en que incurra en ejercicio de sus actividades.; (ii) límite asegurado de $\$ 10.000$ '.000.000 «por evento y en el agregado anual.», y deducible de $\$ 100 ’ .000 .000$; (iii) cláusula «claims made - Base de las indemnizaciones» ...

El reembolso de lo pagado como consecuencia del «Requerimiento especial $\mathrm{n}^{\circ}-000002$ -impuesto de registro- período del 1 al 31 de agosto de 2004».

Una vez cobró firmeza la decisión de la autoridad tributaria territorial, la Cámara de Comercio de Bogotá pagó a favor del Departamento de Cundinamarca en cuantías de $\$ 14.881 .319 .705$ y $\$ 9.669 .716 .840$, los días 21 de octubre de 2015 y 21 de abril de 2016, en su orden, según certificó el Director Financiero de Tesorería de dicho ente, y lo reiteró el subdirector de la misma dependencia, quien informó que «dando cumplimiento a la sentencia proferida por el Consejo de Estado, el 15 de agosto de 2015 la Cámara de Comercio de Bogotá presento (sic) el pago correspondiente al periodo agosto 2004», por los valores y en las fechas que se acaban de indicar.

Tal carga obligacional encuadra en el concepto de «deterioro patrimonial de la asegurada, como consecuencia de los errores y omisiones en que incurra en el ejercicio» de su actividad de «cálculo y recaudo del impuesto de registro», cobertura principal -y extendida- de la póliza 2005-2006, en la que se estipuló un monto límite asegurado de $\$ 10.000$ '000.000, con un deducible por evento de \$100'.000.000.

En ese sentido, la aseguradora deberá indemnizar a su asegurada por la pérdida, pues la misma tuvo origen en la realización del riesgo asegurado. Sin embargo, esa reparación deberá atender los límites de aseguramiento, conforme lo establece el artículo 1079 del estatuto mercantil («El asegurador no estará obligado a responder si no hasta concurrencia de la suma asegurada...»), de manera que la obligación de rembolso se limitará a \$9.900.000.000 (cuantía máxima asegurada, menos el deducible pactado)”.

\section{DECISIÓN:}

En mérito de lo expuesto, la Sala de Casación Civil de la Corte Suprema de Justicia, administrando justicia en nombre de la República y por autoridad de la ley, RESUELVE 
PRIMERO. REVOCAR PARCIALMENTE el ordinal primero de la sentencia que el 31 de enero de 2013 profirió el Juzgado 20 Civil del Circuito de Descongestión de esta . ciudad, en lo concerniente a la denegación de la totalidad de las pretensiones de la demanda del proceso acumulado (rad, 11001-31-03-015-2008-00590-00).

SEGUNDO. DESESTIMAR las excepciones de mérito formuladas por la accionada en la citada causa acumulada, por las razones expuestas, in extenso, en esta providencia.

TERCERO. DECLARAR oficiosamente configurada la excepción de mérito de existencia de deducible, en cuantía de \$100’.000.000.

CUARTO. DECLARAR que el error de la Cámara de Comercio de Bogotá en la determinación del monto del impuesto de registro del período comprendido entre el $1^{\circ} \mathrm{y}$ el 31 de agosto de 2004, está comprendido entre los riesgos asegurados con la póliza $\mathrm{n}^{\circ} 120100000574$.

QUINTO. Como consecuencia de la decisión inmediatamente anterior, SE DECLARA que la demandante sufrió una pérdida patrimonial por valor de DOCE MIL TRESCIENTOS NOVENTA Y CUATRO MILLONES CUATROCIENTOS SESENTA Y UN MIL CINCUENTA PESOS (\$12.394'.461.050).

SEXTO. SE CONDENA a QBE Seguros S.A. a pagar a la Cámara de Comercio de Bogotá la suma de NUEVE MIL NOVECIENTOS MILLONES DE PESOS (\$9.900,000.000), correspondiente al límite del valor asegurado, menos el deducible pactado, a título de indemnización por el acaecimiento del siniestro". ${ }^{13}$

Como puede apreciarse, la Corte aplica el deducible a la suma asegurada y no al valor de la pérdida efectivamente sufrida por el asegurado, desnaturalizando la finalidad del deducible y disminuyendo el monto de la suma asegurada pactada, que como bien lo afirma la Corte en su decisión, es el monto hasta por el cual responde la aseguradora.

Los $\$ 100^{\prime} .000 .000$ del deducible debieron restarse del valor de la pérdida, es decir de los \$12.394'.461.050, lo cual arrojaría una pérdida de \$12.294'.461.050. Tomando en consideración que la suma asegurada pactada fue de $10.000^{\prime} .000 .000$, insuficiente frente a la pérdida sufrida, la aseguradora debió haber sido condenada por ese monto completo, pues el deducible habría sido ya descontado del valor de la pérdida, como correspondería en este caso. Quedaría a cargo del asegurado los primeros $\$ 100$ '.000.000 del deducible y los $\$ 2.294^{\prime} .461 .050$ que exceden de la suma asegurada que corresponden al valor de la obligación de la aseguradora.

\section{BIBLIOGRAFÍA}

Código de Comercio colombiano.

Corte Suprema de Justicia. Sala Civil. SC5217-2019. Diciembre 3 de 2019. Radicación N¹1001-31-03-015-2008-00102-01. Magistrado ponente: Luis Alonso Rico Puerta.

13 Corte Suprema de Justicia. Sala Civil. SC5217-2019. Diciembre 3 de 2019. Radicación Nº11001-31-03015-2008-00102-01. Magistrado ponente: Luis Alonso Rico Puerta. 
Diaz-Granados Ortiz, Juan Manuel. El seguro de responsabilidad. Bogotá D.C.: Editorial Universidad del Rosario y Pontificia Universidad Javeriana, Facultad de Ciencias Jurídicas. $2^{\circ}$ Edición. 2012.

HALPERIN, Isaac. Seguros. Buenos Aires: Ediciones Depalma. 1970.

Laudo Arbitral. Colpensiones vs. AXA Colpatria Seguros S.A. Diciembre 22 de 2020. Árbitros: Arturo Solarte Rodríguez; Juan Carlos Esguerra Portocarrero; Alejandro Venegas Franco.

Martorell, Ernesto Eduardo (Dir). Tratado de Derecho Comercial. 2010. Volumen V. Buenos Aires. Argentina: Editorial La Ley.

NARVÁEz BonnET, Jorge Eduardo. El contrato de seguro en el sector financiero. Tercera edición. Bogotá: Ed. Grupo Editorial Ibáñez, 2014.

Ossa Gómez J., Efrén. Teoría General del Seguro. El Contrato. Segunda Edición actualizada. Bogotá Colombia: Editorial Temis, 1991. 